\title{
Investigation of the Short-Term Effect of Chemonucleolysis with Chondroitinase ABC
}

\author{
Kazutaka YAMADA $^{1) *}$, Shinobu TANABE ${ }^{1)}$, Hiroshi UENO $^{1)}$, Akemi OINUMA $^{2)}$, Toyomi TAKAHASHI ${ }^{2}$, \\ Satoshi MIYAUCHI ${ }^{2)}$, Satoshi SHIGENO ${ }^{3)}$, Tsuneo HIROSE ${ }^{4)}$, Kazuro MIYAHARA ${ }^{5)}$ and Motoyoshi SATO ${ }^{1)}$ \\ ${ }^{1)}$ Department of Veterinary Clinical Radiology, Obihiro University of Agriculture and Veterinary Medicine, Obihiro 080-8555, ${ }^{2)}$ Tokyo \\ Research Institute, Seikagaku Corporation, Tokyo 207-0021, ${ }^{3)}$ Obihiro Neuro-Surgery Hospital, Obihiro 080-0023, ${ }^{4)}$ Research Institute \\ of Information Science Development on Animal Medicine, Obihiro 080-0028 and ${ }^{5}$ Veterinary Teaching Hospital, Obihiro University of \\ Agriculture and Veterinary Medicine, Obihiro 080-8555, Japan
}

(Received 6 September 2000/Accepted 29 January 2001)

ABSTRACT. Chondroitinase ABC (C-ABC) is expected to be a novel agent for chemonucleolysis. The effect of C-ABC was investigated by magnetic resonance (MR) and radiograph. $\mathrm{C}-\mathrm{ABC}$ was administered into the lumbar intervertebral disks on the clinically normal beagles $(\mathrm{n}=5)$, in a dose of $50 \mu \mathrm{l}(12.5$ units as $\mathrm{C}-\mathrm{ABC})$. MR scans were performed pre-dose, and 1, 3, 7, 14 and 28 days after administration of $\mathrm{C}-\mathrm{ABC}$, and the signal intensity (SI) of the nucleus pulposus was measured. Radiographs were taken pre-dose, and 1, 2, 3, 4, 5, 6, 7, 14 and 28 days post-dose, to evaluate narrowing of the disk space in terms of height index (HI). In addition, the quantity of the chondroitin sulfate (CS) and the hyaluronic acid (HA) in the nucleus pulposus were measured by high performance liquid chromatography on day 28 after dosing. SI and HI continuously decreased, following the injection to $37.1 \%$ and $78.9 \%$ of the pre-dose values, respectively. Statistically significant differences $(p<0.01)$ were observed between the C-ABC group and the control group in the respects on day 1 post-dose. CS and HA contents of the nucleus pulposus were noted to be significantly decreased on day $28(p<0.01)$ in the treated group. This agent proved to degenerate proteoglycans in the nucleus pulposus, thus progressively reducing the interdiskal pressure from day 1 post-dose onwards. It is concluded that $\mathrm{C}-\mathrm{ABC}$ is expected to afford its efficacy from early in the course of chemonucleolysis. KEY WORDS: canine, chemonucleolysis, chondroitinase ABC (C-ABC), height index (HI), magnetic resonance (MR).

J. Vet. Med. Sci. 63(5): 521-525, 2001

Intervertebral disk hernia produces neurological deficits, caused by compression to the spinal cord or nerve roots via degeneration of the nucleus pulposus in the intervertebral disk, which is frequently seen in chondrodystrophoid cases. Pharmacotherapy using corticosteroid or surgical intervention like hemilaminectomy is often selected for the treatment. The former does not completely remove the compression while the latter is invasive for the animal. Recently, chemonucleolysis has been applied as a less invasive treatment. Since Smith et al. reported that the chemonucleolysis using chymopapain applied for the first time to an intervertebral hernia case [12], and it was shown to be effective in the clinical setting application as well as in veterinary medicine $[1,10]$. As the use of the proteolytic enzyme chymopapain has been reported to cause the side effects including neurotoxicty, anaphylaxis, subarachoid hemorrhage [2-5], therefore its safety for this purpose remains questionable. Chondroitinase $\mathrm{ABC}(\mathrm{C}-\mathrm{ABC})$ is expected as an alternative agent for chemonucleolysis. It specifically degrades some glycosaminoglycans such as chondroitin sulfate (CS) and the hyaluronic acid (HA). However, previous reports implied that $\mathrm{C}-\mathrm{ABC}$ underwent morphological changes over an extended period post-dose $[9,13]$, our clinical experience with this therapy indicates that clinical manifestations improve in no few cases within one week after administration of C-ABC. This study was

\footnotetext{
* Correspondence to: Yamada, K., Department of Surgical and Radiological Sciences, School of Veterinary Medicine, University of California Davis, CA 95616, U.S.A.
}

conducted to the investigate short-term effect of chemonucleolysis with $\mathrm{C}-\mathrm{ABC}$ by magnetic resonance (MR) and radiograph.

\section{MATERIALS AND METHODS}

Agent for chemonucleolysis: Highly purified pharmaceutical grade C-ABC (Lot. No. BC039P01) was provided by Seikagaku Corporation (Tokyo, Japan) as a mixture with non-ionic iodine contrast agent iohexol (Omnipaque ${ }^{\circledR} 350$, Daiichi Pharmaceutical., Tokyo, Japan) in screw-capped vials $(250$ units $/ \mathrm{m} l)$ per vial and was frozen at $-18^{\circ} \mathrm{C}$ during transport and storage. The $\mathrm{C}-\mathrm{ABC}$ mixture was thawed just before using. The results of a stability test of the $\mathrm{C}-\mathrm{ABC}$ mixture revealed that it remains stable longer than 9 months when stored at $-18^{\circ} \mathrm{C}$ [14]. A phosphate buffer-iohexol mixture was used as the control. Of four consecutive disks (L1-2, L2-3, L3-4 and L4-5), two intervertebral disks were injected with $\mathrm{C}-\mathrm{ABC}$ and the two other disks with the control.

Procedure for chemonucleolysis: Five clinically normal beagles weighing from 9.0 to $13.0 \mathrm{~kg}$ (male, 1-year old) were used in this study. Animals were anesthetized by intravenous injection with thiopental sodium (Ravonal ${ }^{\circledR}$, Tanabe Pharmaceutical, Osaka, Japan) and maintained by halothane (Fluothane $^{\circledR}$, Takeda Chemical Industries, Osaka, Japan) inhalation. The animal was then shaved and disinfected, and placed on an X-ray penetrative animal-holding device, which was inclined at 25 degrees to avoid damaging by a 
spinal needle or contract with the transverse process of the spinal nerve roots. A $70 \mathrm{~mm}, 23$-gauge spinal needle with stylet was inserted into the disk under fluoroscopic control (MMS-155, Hitachi Medical Co., Tokyo, Japan). When the tip of spinal needle reached the anulus fibrosus of the disk, the stylet was pulled out, and a 31-gauge inner needle, which was $7 \mathrm{~mm}$ longer than the stylet, was inserted into the disk itself through the 23-gauge needle [1, 6, 10]. A microsyringe was used to inject $50 \mu l$ of 12.5 units of $\mathrm{C}$ $\mathrm{ABC}$ into the disk. The administration dose is designed by previous report [14]. Radiographs were taken just after the injection to confirm accuracy of the administration, by enhancement of the nucleus pulposus since both chemonucleolysis agent and the control contained iohexol.

Evaluation of MR images: MR images were obtained with a 1.5 tesla magnetic field strength MR unit (VISART $^{\mathrm{TM}}$, Toshiba, Tokyo, Japan). The imaging sequence used was spin echo $(\mathrm{TR} / \mathrm{TE}=2,000 / 80 \mathrm{msec})$, producing $\mathrm{T}_{2}$ weighted images. The slice thickness of each section was $1.5 \mathrm{~mm}$. The scan time of this image was $8 \mathrm{~min} 32$ sec. Animals were placed under anesthesia with thiopental sodium during MR scan. MR scan was performed prior to and at 1, 3, 7, 14 and 28 days after administration of C-ABC. The signal intensity (SI) on region of interest on the nucleus pulposus was measured with a software within the MR unit. The ratio of the SI for non-injected T13-L1 to that at injection site was calculated, and evaluated in terms of percentage to the pre-administration value.

Evaluation of radiographs: The lateral radiographs were taken with X-ray film (HR-3, Fuji Film, Kanagawa, Japan) and an intensifying screen (HR-S, Fuji Film, Kanagawa, Japan). Images were obtained without anesthesia. The Xray examination was performed pre-dose and at 1, 2, 3, 4, 5, $6,7,14$ and 28 days after administration of C-ABC, thereby the narrowing of the disk space was evaluated interms of height index (HI) [6], and expressed as percent of preadministration value.

Biochemical analysis: The nucleus pulposus of L1-2, L23, L3-4 and L4-5 disks were sampled on day 28 after administration of $\mathrm{C}-\mathrm{ABC}$, the samples were analyzed for CS and HA by high performance liquid chromatography [15].

Statistical analysis: MR images, radiographs and biochemical analysis data were assessed for statistical significant differences using the Welch $t$-test.

\section{RESULTS}

Evaluation of MR images: Representative MR images after administration of $\mathrm{C}-\mathrm{ABC}$ are shown in Fig. 1, and changes in SI of the nucleus pulposus observed ( 5 animals, $\mathrm{n}=10$ ) are presented in Fig. 2. The SI of the nucleus pulposus from animals of the administration group was found decreased on day 1 post-dose, and thereafter continued to decrease to $37.1 \%$ of the pre-dose value on day 28 . There were statistically significant differences $(p<0.01)$ in SI between the $\mathrm{C}-\mathrm{ABC}$ group and the control group from day 1 onwards.

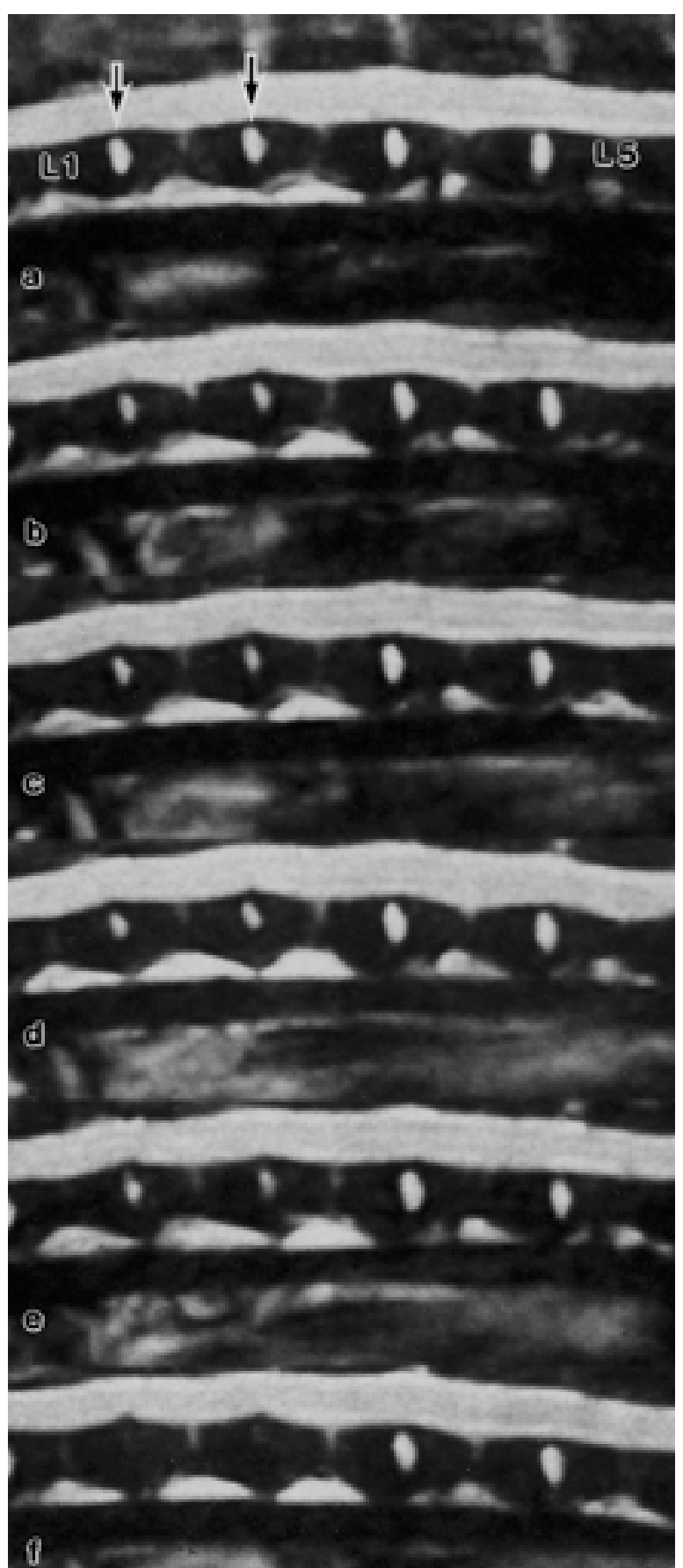

Fig. 1. Representative magnetic resonance (MR) images (spin echo pulse sequence, TR/TE $=2,000 / 80 \mathrm{msec}, 1.5 \mathrm{~mm}$ slice) of the canine lumbar spine after injection of chondoroitinase $\mathrm{ABC}$ (C-ABC). C-ABC injected into the nucleus pulposus of L1-2 and L2-3 (arrows), and the control injected into that of L3-4 and L4-5. (a: pre-dose; b: 1; c: 3; d: 7; e: 14; f: 28 days after administration)

Evaluation of radiographs: Representative radiographs taken after administration of C-ABC are shown in Fig. 3, 


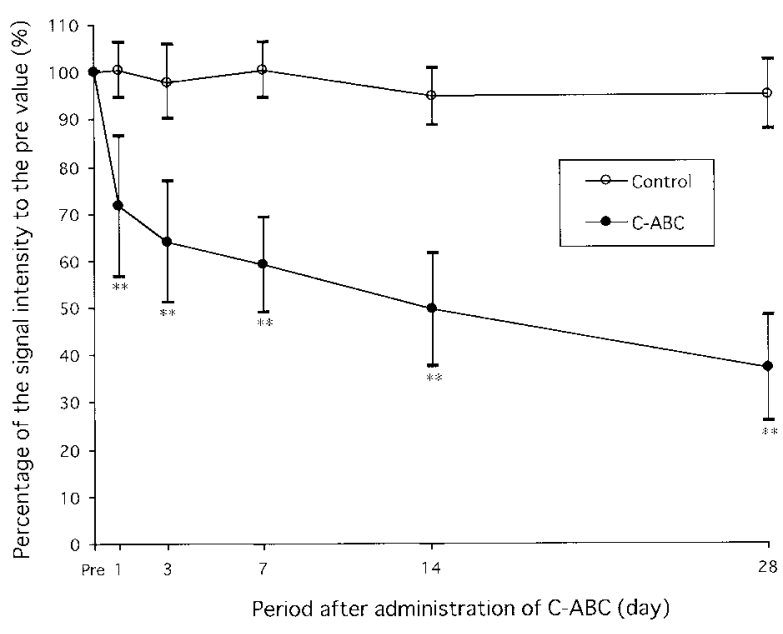

Fig. 2. Changes in signal intensity of the nucleus pulposus (5 animals, 10 disks) on MR images after C-ABC administration (mean $\pm \mathrm{SD}$, Welch $t$-test, $* * P<0.01$ ).

and changes in $\mathrm{HI}$ of the lateral view observed (5 animals, $\mathrm{n}=10$ ) are presented in Fig. 4. The HI in the administration group was noted to be decreased on day 1 , and continued to decrease thereafter to $77.9 \%$ of the pre-dose value on day 28. The statistically significant differences in HI were noted $(p<0.01)$ between the $\mathrm{C}-\mathrm{ABC}$ group and the control group throughout the observation period.

Biochemical analysis: The CS and HA contents of the nucleus pulposus after administration of $\mathrm{C}-\mathrm{ABC}$ on day 28 post-dose are shown in Table 1 . The quantity of CS is $8,602.5 \mathrm{nmol}$ and $135.4 \mathrm{nmol}$ in the control group and the treated group, respectively. The quantity of HA was 164.8 nmol and $20.7 \mathrm{nmol}$ in the control group and the treated group, respectively. The nucleus pulposus CS and HA contents in the treated group were significantly lower $(p<0.01)$ as compared to the control group.

During the entire period of this study, all animals remained free of changes in health status.

\section{DISCUSSION}

We studied the method of imaging evaluation for chemonucleolysis, prior to investigating the effect of C-ABC. At first, MR scanning parameters were settled. It is a well known fact that the contrast of MR image depends on pulse sequence, even if the image is what we call $\mathrm{T}_{2}$ weighted image, and shows subtle changes with pulse sequence. Spin echo pulse sequence, fast spin echo pulse sequence and gradient echo pulse sequence were compared, and the spin echo was selected because it has higher signal intensity of nucleus pulpous and less artifact. Secondly, we compared HI values of the lateral and ventro-dorsal views to seek an appropriate method of evaluation for radiographs. Although the HI decreased in both lateral and ventro-dorsal directions, the variation of ventro-dorsal direction was higher than that of lateral direction. It was possible to render the lumbar

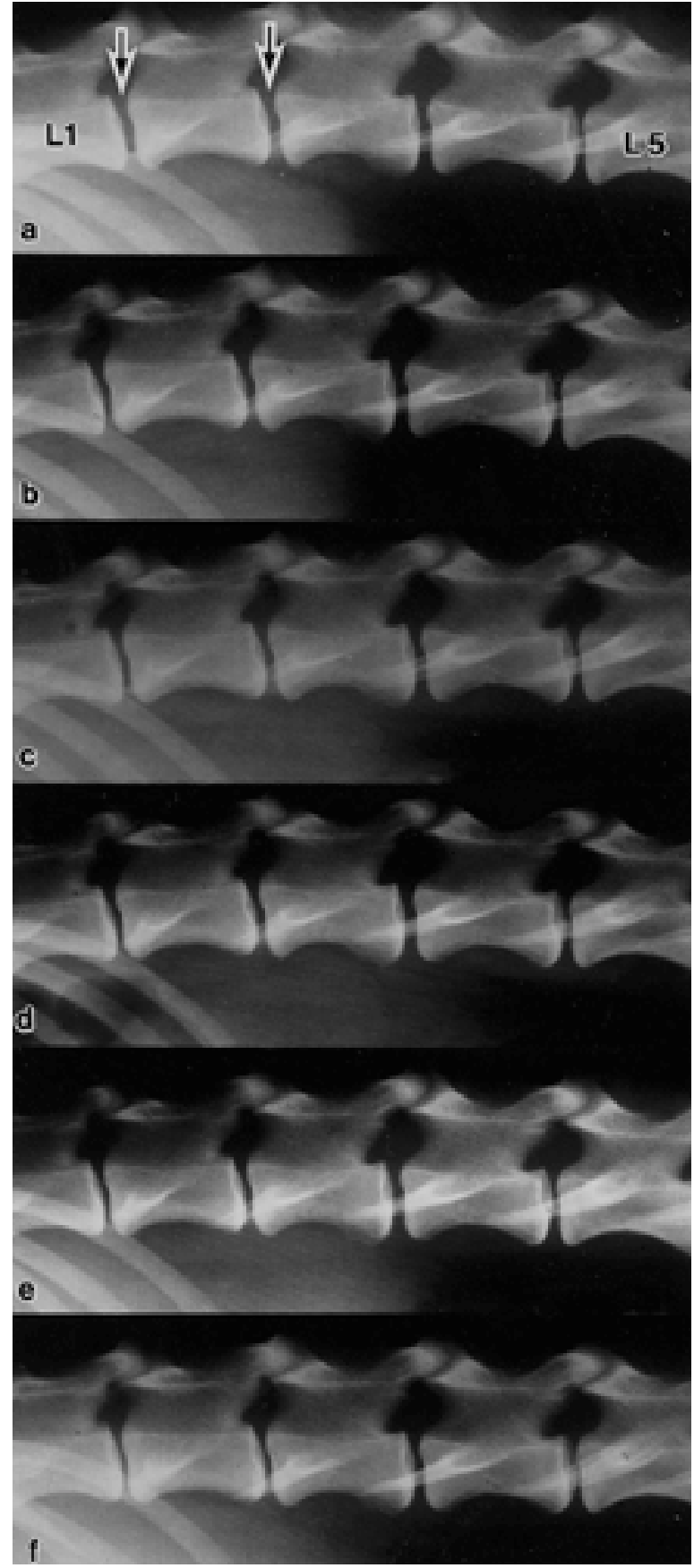

Fig. 3. Representative radiographs of the canine lumbar spine after injection of C-ABC. C-ABC injected into the nucleus pulposus of L1-2 and L2-3 (arrows), and the control injected into that of L3-4 and L4-5. (a: pre-dose; b: 1; c: 3; d: 7; e: 14; f: 28 days after administration)

spine parallel to X-ray film by means of a pad in making lateral projection, but it was hard to keep the lumbar spine parallel in ventro-dorsal view because of the lumbar curvature. So, the lateral HI measurement was used for radiograph 


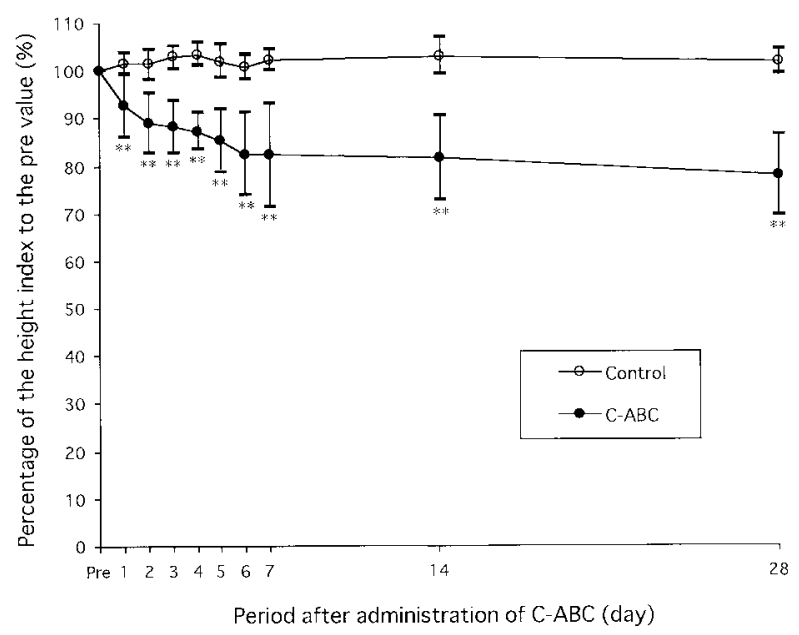

Fig. 4. Changes in height index of the intervertebral disk (5 animals, 10 disks) after $\mathrm{C}-\mathrm{ABC}$ administration (mean $\pm \mathrm{SD}$, Welch $t$-test, ** $P<0.01)$.

Table 1. The chondroitin sulfate and hyaluronic acid contents of the nucleus pulposus after $\mathrm{C}-\mathrm{ABC}$ administration on day 28 post-dose

\begin{tabular}{crrrrr}
\hline \multirow{2}{*}{ Animal\# } & \multicolumn{2}{c}{ Chondroitin sulfate $(\mathrm{nmol})$} & & \multicolumn{2}{c}{ Hyaluronic acid (nmol) } \\
\cline { 2 - 3 } \cline { 5 - 6 } & C-ABC & Control & & C-ABC & Control \\
\hline 1 & 105.1 & $8,400.6$ & & 17.0 & 129.3 \\
2 & 283.6 & $10,055.9$ & & 38.9 & 302.0 \\
3 & 61.0 & $9,893.4$ & & 14.8 & 90.6 \\
4 & 68.0 & $6,041.0$ & & 17.1 & 107.3 \\
5 & 204.4 & $8,612.5$ & & 15.9 & 194.8 \\
\hline mean $(\mathrm{n}=5)$ & $135.4^{* *}$ & $8,602.5$ & & $20.7^{* *}$ & 164.8 \\
\hline
\end{tabular}

(Welch $t$-test, $* * P<0.01$ )

evaluation. The deviation of HI (Fig. 4) might be caused to take radiographs without sedation. It is difficult to evaluate the narrowing of the disk space on MR because of the poor image resolution (the pixel size of about $1.0 \mathrm{~mm}$ ), therefore tracing the intervertebral space might be inaccuracy.

During this research, no changes in healthy condition was noted in any of the animals studied. Therefore, $\mathrm{C}-\mathrm{ABC}$ is thought to be a safe agent for clinical use. C-ABC has been suggested to have fewer side effects than chymopapain, owing to its lack in proteolytic activity and its matrix specifity. Olmarker et al. demonstrated that $\mathrm{C}-\mathrm{ABC}$ caused no changes [7], and reported that no neurologic defect was detected when it affected intrathecal and peripheral nerve tissues [8]. Chemonucleolysis is theoretically not appropriate in the presence of a defect in the anulus fibrosus, because the agent may have the risk of leaking away from the nucleus pulposus through the tear in the anulus fibrosus. While chymopapain has the possibility to damage surrounding tissues if it leaks, $\mathrm{C}-\mathrm{ABC}$ has much less possibility to cause damage even if it is injected not accurately into the nucleus pulposus or in the presence of a defect in the anulus fibrosus (type I herniated disk).
Kudo et al. reported that the effect of chemonucleolysis delayed with aging. The nucleus pulposus undergoes either chondroid or fibrous change with aging [6], so that the present results might be different from previous report [9]. This needs further investigation in the near future.

In this study, continuously decreasing SI on the MR images and $\mathrm{HI}$ on the radiographs were observed. On 28 day post-dose, the progressive decrease in CS and HA contents of the nucleus pulposus was confirmed by the biochemistry analysis. $\mathrm{C}-\mathrm{ABC}$ produced degeneration of proteoglycan in the nucleus pulposus, thereby reducing the intradiscal pressure from day 1 post-dose onwards [11]. It is concluded that $\mathrm{C}-\mathrm{ABC}$ is an alternative agent which is expected to afford the therapeutic effect chemonucleolysis within a short period after dosing.

\section{REFERENCES}

1. Atilola, M. A. O., Morgan, J. P., Bailey, C. S. and Miyabayashi, T. 1988. Canine chemonucleolysis. An experimental radiographic study. Vet. Radiol. 29: 168-175.

2. Cusick, J. F., Ho, K. C. and Schamberg, J. F. 1987. Subarachnoid hemorrhage following chymopapain chemonucleolysis. $J$. Neurosurg. 66: 775-778.

3. Dyck, P. 1985. Paraplegia following chemonucleolysis. A case report and discussion of neurotoxity. Spine 10: 359-362.

4. Egro, H. 1983. Transverse myelitis following chemonucleolysis; Report of a case. J. Bone. Joint Surg. [Am] 65: 1328-1330.

5. Hall, B. B. and McCulloch, J. A. 1983. Anaphylactic reactions following the intradiscal injection of chymopapain under local anesthesia. J. Bone Joint Surg. [Am] 65: 1215-1219.

6. Kudo, T., Sumi, A. and Hashimoto, A. 1993. Experimental chemonucleolysis with chymopapain in canine intervertebral disks. J. Vet. Med. Sci. 55: 211-215.

7. Olmarker, K., Danielsen, N., Nannmark, U., Sennerby, L. and Rydevik, B. 1990. Microvascular effects of chondroitinase $\mathrm{ABC}$ and chymopapain. An in vivo experimental study on hamsters and rabbits. Clin. Orthop. 257: 274-279.

8. Olmarker, K., Danielsen, N., Nordborg, C. and Rydevik, B. 1991. Effects of chondroitinase ABC on intrathecal and peripheral nerve tissue. An in vivo experimental study on rabbits. Spine 16: 43-45.

9. Ono, A., Harata, S., Takagaki, K. and Endo, M. 1998. Proteoglycans in the nucleus pulposus of canine intervertebral discs after chondroitinase ABC treatment. J. Spinal. Disord. 11: 253-260.

10. Saunders, E. C. 1964. Treatment of the canine intervertebral disc syndrome with chymopapain. J. Am. Vet. Med. Assoc. 145: 893-896.

11. Sasaki, M., Takahashi, T., Miyahara, K. and Hirose, T. 2001. Effects of chondroitinase $\mathrm{ABC}$ on intradiscal pressure in sheep: An in vivo study. Spine 26: 463-468.

12. Smith, L., Garvin, P. J., Gesler, R. M. and Jennings, R. B. 1963. Enzyme dissolution of the nucleus pulposus. Nature (Lond.) 198: 1311-1312.

13. Sugimura, T., Kato, F., Mimatsu, K., Takenaka, O. and Iwata, H. 1996. Experimental chemonucleolysis with chondroitinase ABC in monkeys. Spine 21: 161-165.

14. Takahashi, T., Nakayama, M., Chimura, S., Nakahara, K., Morozumi, M., Horie, K., Fujita, Y. and Hirose, T. 1997. Treatment of canine intervertebral disc displacement with 
chondroitinase ABC. Spine 22: 1435-1439.

15. Toshida, K., Miyauchi, S., Kikuchi, H., Tawada, A. and Tokuyasu, K. 1989. Analysis of unsaturated disaccharides from gly- cosaminoglycan by high-performance liquid chromatography. Anal. Biochem. 177: 327-332. 